SLAC-PUB-9007

September 27, 2001

\title{
RADIATION SAFETY SYSTEMS FOR ACCELERATOR FACILITIES*
}

\author{
James C. Liu ${ }^{1}$, Jeffrey S. Bull ${ }^{2}$, John Drozdoff ${ }^{3}$, Robert May ${ }^{4}$, and Vaclav Vylet ${ }^{5}$
}

1. Stanford Linear Accelerator Center, MS48, P.O. Box 4349, Stanford, CA 94309, USA

2. LANSCE, Los Alamos National Lab, Los Alamos, NM 87545, USA

3. TRIUMF, 4004 Westbrook Mall, Vancouver, B. C., Canada, V6T2A3

4. TJNAF, Jefferson Lab., 12000 Jefferson Ave. MS12A, Newport News, VA 23606, USA

5. DFELL, OESO/DUMC Box 3155, Duke University, Durham, NC 27710, USA

\begin{abstract}
The Radiation Safety System (RSS) of an accelerator facility is used to protect people from prompt radiation hazards associated with accelerator operation. The RSS is a fully interlocked, engineered system with a combination of passive and active elements that are reliable, redundant, and fail-safe. The RSS consists of the Access Control System (ACS) and the Radiation Containment System (RCS). The ACS is to keep people away from the dangerous radiation inside the shielding enclosure. The RCS limits and contains the beam/radiation conditions to protect people from the prompt radiation hazards outside the shielding enclosure in both normal and abnormal operations. The complexity of a RSS depends on the accelerator and its operation, as well as associated hazard conditions. The approaches of RSS among different facilities can be different. This report gives a review of the RSS for accelerator facilities.
\end{abstract}

Submitted to the Journal of Radiation Protection and Dosimetry

*Work supported by Department of Energy contract DE-AC-03-76SF00515 


\section{INTRODUCTION}

The prompt radiation hazards associated with the accelerator operation can be high. The primary goal of the Radiation Safety System (RSS) of an accelerator facility is to protect people from prompt radiation hazards with a fully interlocked, engineered passive/active system that is reliable, redundant, and fail-safe. The RSS can generally be divided into two complementary sub-systems: the Access Control System (ACS) and the Radiation Containment System (RCS). The ACS is to keep people from being inside the shielding enclosure, where the dangerous, and often lethal, prompt radiation exists. On the other hand, by containing and/or limiting both the beam and radiation conditions, the RCS protects people from the potential prompt radiation hazards outside the shielding enclosure under both normal and abnormal accelerator operation conditions.

The complexity of a RSS depends on the accelerator characteristics, how the facility is operated, and its radiation hazard conditions. Currently the policies and approaches of RSS among facilities are different. This report gives a review of the RSS for five North American high-energy accelerator facilities. The RSS of the B-Factory accelerator facility at Stanford Linear Accelerator Center (SLAC) is described in more detail to illustrate the general principles and practices of a RSS. The main elements of the RSS of Los Alamos Neutron Science Center (LANSCE), Tri-University Meson Facility (TRIUMF), Thomas Jefferson National Accelerator facility (TJNAF), and Duke Free Electron Laser Facility (DFELL) are reviewed to show the similarity and contrast of RSSs among facilities. The references ${ }^{(1-8)}$ can be consulted for more details of the RSS descriptions at these facilities.

\section{ACCELERATOR FACILITY INTRODUCTION}

\section{B-Factory}

Figure 1 shows a schematic layout of the B-Factory, the latest and the largest accelerator facility at SLAC, and its associated safety systems. The positron beam with a maximum allowed energy of $4 \mathrm{GeV}$ is extracted at sector 4 of the 2 mile long LINAC, whereas the electron beam with a maximum allowed energy of $12 \mathrm{GeV}$ is extracted at sector 10. Further downstream in the Beam Switch Yard (BSY) area, the low-energy south injection transport line (LESIT) takes the positron beam into the low-energy storage ring (LER), while the high-energy north injection transport line (HENIT) takes the electron beam into the high-energy storage ring (HER). The LER is positioned $1 \mathrm{~m}$ above the HER in an underground ring tunnel of $2200 \mathrm{~m}$ circumference (called the PEP-II ring). There are five large Interaction Region (IR) halls (each hall is about $20 \times 16 \mathrm{~m}^{2}$ and $15 \mathrm{~m}$ high) around the ring, which workers may occupy. After injection, the stored electron and positron beams circulate in opposite directions and collide at the 
Interaction Point inside the BaBar detector located at one of the IR. These IR halls have $1.2 \mathrm{~m}$ thick concrete roofs and $1 \mathrm{~m}$ thick concrete walls, separating the beamlines from the occupied area. Since most of the ring is covered by at least a thickness of $5.5 \mathrm{~m}$ of earth, these IRs have the weakest part of the shield.

\section{LANSCE}

Figure 2 shows schematically the LANSCE accelerator and its safety systems. The main accelerator is the $800-\mathrm{MeV}$ proton linear accelerator, which can provide up to $1 \mathrm{~mA}$ of $\mathrm{H}^{+}$beam and 150 $\mu \mathrm{A}$ of $\mathrm{H}^{-}$beam for the experimental areas. Area A is used for high-intensity (up to $1 \mathrm{~mA}$ ), high-power beam experiments. Line $\mathrm{X}$, limited to $10 \mu \mathrm{A}$ beam current by a pair of toroids, is used for proton radiography (Line C) and ultra-cold neutron experiments (Line B). Line D transports beam to the Manual Lujan Neutron Scattering Center (maximum beam150 $\mu \mathrm{A}$ ), a high intensity neutron spallation source, and the Weapons Neutron Research area (maximum beam $5 \mu \mathrm{A}$ ). Here, experimental areas include a generalpurpose proton experimental area (Target 2) and a broadband neutron source (Target 4). The Proton Storage Ring is a beam accumulator used in conjunction with the spallation neutron source. Due to its thinner shield, Line D uses current limiting toroids to ensure that the beam power limits are not exceeded

\section{TRIUMF}

Figure 3 shows the TRIUMF cyclotron vault and the upstream sections of three primary beamlines 1,2 , and $4{ }^{(6)}$. The $500-\mathrm{MeV}$ cyclotron can accelerate up to $250 \mu \mathrm{A}$ of $\mathrm{H}^{-}$ions to a maximum energy of $500 \mathrm{MeV}$. Protons can then be extracted into four different beamlines (only three are shown here) simultaneously with two of the four beamlines splitting up into multiple branch lines. Beamlines $1 \mathrm{~A}$ and $2 \mathrm{~A}$ are high intensity beamlines (proton currents up to $200 \mu \mathrm{A}$ and $100 \mu \mathrm{A}$, respectively). Other beamlines are of low intensity, up to $1 \mu \mathrm{A}$. The different beamlines and branch lines are shielded for different currents and beam loss scenarios.

\section{TJNAF}

TJNAF has a $4 \mathrm{GeV}, 200 \mu \mathrm{A}$ (design values) electron beam accelerator for experimental nuclear physics and a $50 \mathrm{MeV}, 100 \mathrm{~mA}$ (design values) energy recovery electron accelerator to drive a free electron laser. Figure 4 shows that the $4 \mathrm{GeV}$ injector can inject the beam through the switch yard to three experimental halls with variable beam current and energy (up to design energy). Access is allowed to areas adjacent to locations where beam delivery is ongoing. Passive shielding, access controls, and safety 
systems that verify proper configuration and function of safety devices, along with auxiliary radiation monitors, are used to ensure personnel safety.

\section{DFELL}

DFELL has three accelerator areas; Linac, storage ring, and a gamma line and gamma vault. The Linac and ring can only be operated together. The ring can be operated in normal mode (generating UV light for experiments) or gamma mode (requiring special bunching and timing of stored beam to create high-energy gammas, which are channeled into the gamma vault for experiments). Currently the maximum energy is $300 \mathrm{MeV}$ for the Linac and $1 \mathrm{GeV}$ for the ring (achieved through RF cavity acceleration in the ring).

\section{ACCESS CONTROL SYSTEM (ACS)}

To facilitate accelerator operation and access control, the B-Factory is divided into three major areas (called Personnel Protection System, PPS, areas): the LINAC, the BSY, and the PEP-II ring. Through the use of ACS, personnel can safely occupy one or more PPS areas while the remaining areas (generally the upstream ones) have radiation and/or electrical hazards due to accelerator operation. The 10CFR835 ${ }^{(9)}$ requires that the access control devices shall function automatically (or the area be locked) to ensure that no one is inside a High Radiation Area where a worker could receive more than $10 \mathrm{mSv}$ in any one hour. Thus, it is clear that the beamline areas of the B-Factory require the use of an interlocked ACS.

The ACS consists of three elements: beam inhibiting devices, shielding barrier (i.e. concrete structure for accelerator housing), and an entry module at each entrance of a PPS area. The beam inhibiting devices are interlocked safety devices that remove beam and hazards in the occupiable PPS areas (e.g. interlocked power supplies for the high voltage to the gun and modulators; beam stoppers in the beamline between two adjacent PPS areas). The shielding housing itself also serves as a physical barrier, which makes the access to a hazardous PPS area possible only through the entry points. The shielding aspect will be discussed in the RCS section.

At SLAC, there are generally four access states for a PPS area:

1) Permitted Access: This state allows unlimited and uncontrolled entry, and both the radiation and electrical hazards are interlocked to be off.

2) Controlled Access: This state allows limited and controlled entry, and both the radiation and electrical hazards are interlocked to be off.

3) Restricted Access: This state allows no entry, and only the radiation hazard is interlocked to be off. 
However, under a special Restricted Access Safety Key mode in this state, workers are allowed to enter a PPS area with an electrical hazard in order to perform special tests.

4) No Access: This state allows no one in a PPS area, and both the radiation and electrical hazards can be on.

\section{Entry Module}

Typical features of an entry module for a PPS area, illustrated in Figure 5, include:

1) An interlocked, and lockable, outer door (with emergency entry and exit).

2) A maze and an interlocked, but not locked, inner gate.

3) A keybank with keys. In the Controlled Access or Restricted Access state, everyone entering the area is required to take a key from the keybank and carries it with him/her during the period of access.

4) A key switch and push button for door release (controlled by operator).

5) An access and beam status display, as well as radiation warning light and sign.

6) Intercom or telephone for communication with operator.

7) TV camera to facilitate access control by operator.

The above features allow the accelerator operators to maintain access control and allow people a safe entry. A search of a PPS area by the operators, following a well-defined procedure and routes to clear people away before operation, is required after the area has been in a Permitted Access state. People are also warned of potential beam hazard prior to operation by dimmed lights and audible warnings. The emergency-off push buttons and the emergency entry/exit are two features that allow people to be able to respond to dangerous beam situations if they are accidentally left inside a PPS area. Most features above are required by NCRP ${ }^{(10)}$ and are standard at most large accelerator facilities.

\section{Beam Inhibiting Device (Beam Stopper)}

The beam inhibiting devices are specific accelerator or beam line components that are used as interlocked safety devices to keep an area safe for access by removing hazards or containing the radiation hazard elsewhere. Examples of beam inhibiting devices include, but are not limited to, RF or modulator power supplies, guns, and beam stoppers. A beam stopper can be either a physical device that can be inserted into the beamline to block the beam or an electromagnetic dipole in the beamline to render the beam transport impossible. 


\section{B-Factory}

It is required at SLAC to have at least two, but typically three, beam inhibiting devices for a primary beamline (see Figure 5). If only two beam stoppers are used, the beam cannot be parked on the first stopper unless a prior failure occurs. The types and locations of the beam stoppers obviously have implications on the dose rate downstream where workers could be present. At SLAC, there are certain shielding design requirements that a beam stopper system must also meet (see the Shielding section below).

Figure 1 shows the three PPS areas (LINAC, BSY, and PEP-II ring) and associated beam stopper systems at the B-Factory. For example, there are three LESIT transport beam stoppers (two physical devices, ST9049 and ST9155, and the dipoles, B2-B4) to keep the PEP-II ring safe for access while the positron beam remains in BSY. To stop the LER from running, the beam inhibiting devices are two physical devices ST2018 and ST2019 in the ring, RF cavities and bends of the ring.

To meet the fail-safe requirement, it is required at SLAC that the mechanical stoppers (beam stopper, dump and protection collimator) be equipped with burn-through monitors and protected by at least two other electronic devices (ion chambers or temperature sensors), if a radiation hazard to personnel from the potential damage of beam stoppers due to the maximum credible beam power is possible. For example (see Figure 1), the beam can be parked on the first LESIT physical beam stopper ST9049 in normal operation. Thus the stopper is water-cooled (monitored by a flow switch) to withstand a power of $30 \mathrm{~kW}$ and is also protected by two average current monitors (I88 and I90) upstream, which limit the power of beam that can hit the beam stopper. The last LESIT physical beam stopper ST9155 has a power rating of $0.5 \mathrm{~kW}$ and can be hit by beam only when the first two beam stoppers fail. The stopper ST9155 is protected by a pair of protection ionization chambers, which are set to sense the radiation and trip the beam off if the stopper is hit by more than $0.1-\mathrm{kW}$ beam. On the other hand, the bends B2-B4 need not be protected. This is because, even if they are hit and damaged by beam, the beam still cannot be transported and the area downstream remains safe.

When there is an ACS violation (e.g., crashing in through the ACS door or pushing an emergency-off button inside the ring), the interlocked response is that the voltage supplies for the LINAC klystron modulators, the ring bends, and RF cavities will all be turned off to remove the beam hazards immediately. In addition, the HENIT and LESIT transport beam stoppers and the HER and LER ring stoppers will also function automatically (i.e. in or off).

\section{SSRL}

Figure 6 shows the beam stopper system at SSRL (a synchrotron radiation facility at SLAC). The discussion here will concentrate on the RSS for synchrotron radiation beamlines, since this is the part that 
is unique and different from other types of accelerator facilities. In each main synchrotron radiation beamline, there are two injection stoppers to block the injection beam when the beam is mis-steered into the synchrotron beamline during injection. The water-cooled movable mask absorbs the intense synchrotron radiation, thus protecting the two injection stoppers. The movable mask and the two injection stoppers in every main beamline are interlocked to be in when the injection septum of the storage ring is on.

The beam stoppers to block the gas bremsstrahlung and synchrotron radiation from going to the X-ray hutch (an experimental station) are two mechanical devices (called hutch shutters) in the X-ray branch line. The two hutch shutters are interlocked to be in when the hutch is in the Permitted Access state. There is also one fixed beam stop at the end of the X-ray hutch or in the median plane of the VUV line to block the gas bremsstrahlung. Similar to the function of hutch shutters for the $\mathrm{x}$-ray line, there is one (or two) beam shutter(s) followed by an isolation valve for each VUV branch line.

Synchrotron radiation accelerator facilities are low-power $(<100 \mathrm{~W})$ facilities. Therefore, the RCS is less complex in that there is no need to protect the shielding and beam stoppers from potential radiation damage. For example, there is no cooling water, burn-through monitor, or ion chamber attached to, and to protect, any local lead shielding or beam stoppers that are used to contain the beam. However, due to the high power of the synchrotron radiation, the devices that contain or absorb the synchrotron radiation in the beamlines (e.g., masks, hutch shutters, and beam stop) must be water-cooled (flow switches interlocked) and/or equipped with burn-through monitors.

\section{LANSCE}

Two beam stoppers are required to make a downstream area safe. Beam plugs are the usual mechanical beam stoppers, although in one instance, a bending magnet with a power supply off condition is used. Four types of beam stoppers, including the final beam stops and targets, are shown in Figure 2. Run Permit plugs are part of the interlock system designed to protect equipment and, thus, it is not considered a safety system. Dual-use beam plugs are part of both the Run Permit and the RCS systems. They physically isolate downstream beam transport areas for entry and maintenance. Only those plugs designated as "run-mode endpoints" can handle the full power of the beam. The non-endpoint beam plugs are protected from inadvertent beam impingement by the Run Permit protection system. Other run-mode endpoints where the beam can be parked are the stopping targets and the various non-target beam stops. No radiation detectors are attached to the beam stoppers.

The two sets of beam stoppers positioned in the LINAC are fusible beam plugs (these are RSS plugs). These fusible beam plugs have a thin window before the physical beam blocker. The space between the window and the blocker is open to the atmosphere. If the beam impinges on one of these 
plugs, it burns through the window and lets the accelerator up to atmospheric pressure. The beam cannot propagate through air, thus the accelerator is shut down. However, other systems normally shut off the beam first, making these fusible beam plug devices the final safety barrier. One pair of these fusible beam plugs is automatically inserted if a fault is detected in the RSS.

\section{$\underline{\text { TRIUMF }}$}

Three beam inhibiting devices (with at least one beam stopper) are required. Figure 3 shows that each main beamline has three beam inhibiting devices (stripper OUT, blocker IN and dipole OFF) that are used to define that the beamline and all its associated exclusion areas as being 'OFF' and accessible.

No burn-through plug, flow switch or temperature sensor is attached to protect the beam stoppers. However, discrete radiation detectors are positioned near the beam stoppers (blocker and dipole) to terminate the beam if the stoppers are hit by beam. This beamspill monitoring system consists of 55 highlevel detectors (scintillation detector and ion chamber), all of which are located inside exclusion area shielding close to the accelerator and all beamlines. They are used even in locations (such as the vault) where a beam loss would not cause significant radiation fields outside shielding. The 'trip' setpoint for each detector is based on normal beam losses and the 'time-to-trip' is $200 \mathrm{msec}$.

Currently no evaluation of the potential hazard in the cases of multiple (two out of three) stopper failures is made. However, TRIUMF is attempting to utilize only those beam stopper device combinations that do not generate significant hazards even in the event of multiple device failures. This is being accomplished by using three different safety devices in each primary beamline in the cyclotron vault area, rather than in the downstream branch line areas. The cyclotron vault is shielded for the total loss of maximum approved beam power whereas the downstream beamline areas are not.

\section{TJNAF}

The ACS at TJNAF uses three independent beam inhibiting devices with at least two different technologies (magnet power supplies, beam stopper, etc). Any beam inhibiting device which can intercept the beam must a) be able to fully absorb the maximum credible beam power indefinitely, or b) be equipped with auxiliary devices (beam diffuser and current monitor) which terminate beam delivery within a time short enough that the beam power absorbing capability of the beam intercepting device is not compromised. The ACS is based on programmable logic control (PLC) and uses redundant systems as part of the overall design ${ }^{(7)}$. 


\section{DFELL}

If the Linac or ring is to be accessed, the beam is interlocked to be off by removing the RF to both the Linac cavities and the gun. There are no beam stoppers between the ring and the gamma vault. There is, however, a radiation detector, positioned on top of the gamma line inside the ring, which is interlocked to detect and terminate any accidental gamma mode operation. There is also a special and separate keybank panel that has to be activated for gamma mode operation using a key that is normally locked and kept in a keysafe. In addition, operators, who are required administratively not to run the ring in the gamma mode, have to take some efforts to change from normal mode to gamma mode.

For the electron beam to accidentally go into the gamma line, a simultaneous short of both coils of the first dipole in the east arc has to occur. In case the shorting occurs, there is a permanent magnet located at the exit of the gamma line inside the ring to deflect the beam downwards. There is a reed magnetic field monitor continuously monitoring the presence of the permanent magnet. The reed field monitor is interlocked such that the RF is not enabled if the permanent magnet is removed.

\section{RADIATION CONTAINMENT SYSTEM}

Radiation containment is generally accomplished by a combination of passive systems (shielding, protection collimator, etc.) that attenuate/intercept radiation and active systems (electronic monitors) that terminate radiation hazards when out-of-tolerance conditions are detected. The main system elements of the RCS are power limiting devices, shielding, and active radiation monitoring devices (see Figure 5). The beam stopper system described in the ACS section is treated as a system element of RCS in some facilities.

\section{Power limiting device}

\section{B-Factory}

Three beam power levels have to be considered for shielding design at SLAC: normal beam power, allowed beam power, and maximum credible beam power. Table 1 lists the corresponding levels considered for the PEP-II ring. The facility runs at normal beam power most of the time and occasionally runs at the allowed beam power. Under extreme tuning conditions, the LINAC is capable of injecting the maximum credible beam power to the ring. SLAC requires that a minimum of three devices be used to limit the injection beam current below the allowed beam power. For example, three average current monitors (toroids I88, I90, and I91 in LESIT line in Figure 1) are used to limit the positron beam current. 
There is also a meter relay interlock measuring the magnetic field strength of the dipole B1 to ensure that the positron beam energy is within the allowed energy range.

\section{LANSCE}

LANSCE uses two current limiting devices on a low-power beamline (e.g., lines X and D) to limit the beam power to the maximum allowed beam power. Each current limiter has one current toroid (whose function is monitored and tested continuously and automatically) and redundant signal processing and relay circuitry. Although each individual current limiter meets the fail-safe and redundant requirements, they are installed in pairs. The locations of the current limiters are shown in Figure 2. The LINAC is incapable of accelerating protons to energies higher than $800 \mathrm{MeV}$ and, therefore, no interlocked devices are used to limit the beam energy.

\section{TRIUMF}

There are no current or energy limiting devices, although the cyclotron is capable of delivering power higher than the rated (i.e., maximum approved) power of any individual beamline. Instead a system of discrete radiation detectors (plastic scintillators or ion chambers) positioned close to beamlines throughout the facility are used to monitor and limit excess current or wrong energy of the beam. It is an administrative requirement that the operators run the beam below the rated beam power and the response and interlock of these detectors are tested manually about twice per week.

\section{TJNAF}

The basic current limiting element is a three-port resonant cavity capable of measuring the beam current. Two ports of the current measuring device provide redundant beam current signals to the A and B chains, while the third port provides a means to manually calibrate and verify the functionality of the current monitoring hardware.

One three-port resonant cavity is used in the injector. There is also one three-port resonant cavity located immediately upstream of each beam stopper in the switch yard upstream of experimental halls to ensure that the beam stopper is operated within its power and current handling capabilities.

\section{DFELL}

There are no interlocked energy or current limiting devices, but the energy and current are limited by facility hardware capabilities. For example, the power supply of cathode heater current does not allow a fully thermionic mode operation, which could generate a higher beam current. In the photocathode mode, the laser is already operated at its physical maximum power and frequency. The above hardware 
limits correspond to a maximum average beam power of $0.21 \mathrm{~W}$ at $270 \mathrm{MeV}$, though the typical injection power is only $0.01 \mathrm{~W}(270 \mathrm{MeV}, 54 \mathrm{pC} /$ pulse, at $2 / 3 \mathrm{~Hz})$.

\section{Shielding}

\section{B-Factory}

At SLAC, three shielding design criteria are used for normal and abnormal (mis-steered and system-failure) beam loss situations:

1) The annual dose outside the shielding from normal beam losses (conservatively estimated by accelerator physicists), including operations at both the normal and allowed beam powers, is no more than $10 \mathrm{mSv}$ ( $1 \mathrm{mSv}$, if the area is occupied frequently by non-radiation workers). This is also a Department of Energy limit.

2) The dose rate outside the shielding from mis-steered beam loss at the allowed beam power level is no more than $4 \mathrm{mSv} \mathrm{h}^{-1}$ (this is only a SLAC guideline). However, if there are beam loss monitors near the beamline to terminate excess beam losses, the limit can be raised or removed. This is an example of using active devices to supplement passive shielding for abnormal beam losses.

3) The radiation level outside the shielding from any system-failure situation is no more than $250 \mathrm{mSv} \mathrm{h}^{-}$

${ }^{1}$. This assumes that the event can be terminated within a short period of time, e.g., a few minutes, by human intervention. However, the resulting dose rate can be allowed to be higher than $250 \mathrm{mSv} \mathrm{h}^{-1}$, if the event can be terminated by engineered means (e.g., the beam burns through vacuum chamber resulting in vacuum failure), within a short time such that the integrated dose per event is no more than $30 \mathrm{mSv}$. System-failure situations refer to the cases in which there is at least one interlocked device failure. An example of a system-failure situation is that when two out of three beam stoppers fail and the beam is still delivered at the allowed beam power. For example (see Figure 1), when the HENIT ST6049 fails to be in and bends B2-B9 fail to be off, a $3.5 \mathrm{~kW}$ electron beam hits the last stopper ST6155. The resulting dose rate in the ring downstream of the ACS gate is still less than 250 $\mathrm{mSv} \mathrm{h}^{-1}$. Another system-failure situation is that when all three average current monitors fail and the beam with maximum credible beam power is delivered and lost fully at a point. One example for this case is that the toroids I58, I60 and I61 fail and a 333-kW electron beam is delivered to hit the vacuum chamber in an IR. The resulting dose rate outside the IR concrete wall will be higher than $250 \mathrm{mSv} \mathrm{h}^{-1}$. However, in this high power case, it has been shown that the vacuum chamber will be burned through within seconds. Therefore, the injection beam cannot be sustained for long and, thus, the integrated dose per event is less than $30 \mathrm{mSv}$. It has also been found that the power threshold of the vacuum chamber burn-through is $110 \mathrm{~kW}$. At this power level, the dose rate outside an IR 
concrete wall is less than the limit of $250 \mathrm{mSv} \mathrm{h}^{-1}$. Note that at SLAC the case of all three beam stoppers failing, as well as simultaneous failures for both the beam stopper and average current monitor systems, is considered an incredible event and need not be considered.

SLAC also requires that, for each facility, the shielding design needs to ensure that the maximum annual dose to the general public living near the SLAC boundary from skyshine radiation is no more than $0.1 \mathrm{mSv} \mathrm{y}^{-1}$.

\section{LANSCE}

LANSCE shielding is dominated by the concept of a mis-steered beam loss at a point (with the maximum allowed beam power limited by toroids) and the shielding design limit for this case is $250 \mathrm{mSv}$ $\mathrm{h}^{-1}$. Fail-safe gamma-ray detectors located inside the beam line enclosure are used to detect beam spills in Line D. These detectors are part of the RCS, and automatically insert a pair of fusible beam plugs if the radiation levels exceed a predetermined threshold. Other radiation detectors, both inside and outside the beam tunnel, are part of accelerator protection systems (Fast Protect and Run Permit systems). These are not safety-rated systems, i.e., not fail-safe and/or redundant. No other devices are used to terminate missteered beam events.

Credit is taken for the active toroids and the power-related configuration control program so that the failure of toroids and the loss of beams with higher powers are deemed incredible. Credit for the beam stoppers is also taken and, thus, no evaluation of the potential hazard for the failure of stoppers is performed. Therefore, the system failure situations used at SLAC are not considered in the LANSCE shielding design.

For normal beam losses, the shielding and access control perimeters are established to support LANL requirements (designed to $20 \%$ of the established standards) and ALARA objectives.

Shielding is also designed so that no offsite exposure to a person from the Design Basis Accident (mis-steering of the beam and failure of radiation monitors) exceeds $10 \mathrm{mSv}$ in one hour. In normal operation, air dose is the main source and is limited to $0.01 \mathrm{mSv} \mathrm{y}^{-1}$. Skyshine radiation is estimated to be trivial and no limit specific to skyshine is set in the Design Basis Accident.

\section{TRIUMF}

For normal beam losses, the shielding design limit is $0.01 \mathrm{mSv} \mathrm{h}^{-1}$. Occupancy factors are used to ensure that workers and public receive less than their legal annual dose limits. For abnormal beam losses, the policy is to consider the worst-case credible beam loss scenario, which is a full, instantaneous beam loss at a point with the maximum approved beam power and the dose limit outside shielding is $1 \mathrm{~Sv} \mathrm{~h}^{-1}$.

Radiation detectors terminate any abnormal beam losses fast enough so that the integrated dose 
per event (under rated beam power conditions) is less than $10 \mathrm{mSv}$. Two independent radiation detection systems (part of the RSS) back up each other in areas where abnormal beam losses may lead to significant dose rates outside shielding: the beamspill detector system described in the beam stopper section and the neutron monitors described in the ARMD section below.

The shielding design limit for TRIUMF boundary dose is $0.05 \mathrm{mSv}^{-1}$ from all pathways, including air, direct, and skyshine.

\section{TJNAF}

The design of passive shielding in and around the accelerator and the experimental halls is matched to beam losses expected from normal accelerator operation within the safety envelope (limit of $2.5 \mathrm{mSv} \mathrm{y}^{-1}$ ). Design considerations and limits for abnormal beam losses (in both mis-steered and systemfailure scenarios) is less than $0.15 \mathrm{~Sv} \mathrm{~h}^{-1}$, and the event should be terminated within $1 \mathrm{~s}$ (ensured by critical devices in the RSS).

\section{DFELL}

There is no internal shielding design policy, but the annual dose limits of the State regulation (50 $\mathrm{mSv} \mathrm{y}^{-1}$ for radiation workers, $1 \mathrm{mSv} \mathrm{y}^{-1}$ for non-radiation workers, and $1 \mathrm{mSv} \mathrm{y}^{-1}$ for general public) are observed in the shielding design and verification practice for both workers and general public.

\section{Active Radiation Monitoring Device (ARMD)}

The ARMDs are needed to detect and terminate the radiation hazards outside the shielding from either the mis-steered or the system-failure beam loss situations.

\section{B-Factory}

At least one pair of monitors consisting of a neutron detector and a photon detector is mounted outside every IR concrete wall. They are interlocked to terminate the beam if the preset trip level $(0.1$ $\mathrm{mSv} \mathrm{h}^{-1}$ for occupied areas) is exceeded or the instrument has a failure, e.g., a power supply fails or a detector probe is not working. The ARMDs also have a warning level set at $0.05 \mathrm{mSv} \mathrm{h}^{-1}$. Each photon detector is self-tested by a built-in photon source and each neutron detector is self-tested by the cosmicray neutrons.

SLAC also uses interlocked devices (called beam loss monitors) inside the beamline enclosure to monitor and terminate beam losses. Such devices can be a pair of current monitors (or a pulse-to-pulse comparator) positioned at both ends of the beamline or radiation sensors positioned along the beamline. 
These devices can complement, but do not replace, the functions of ARMDs.

\section{LANSCE}

LANSCE has three ARMD systems, which utilize both gamma detectors in the beam tunnels and neutron detectors outside the shielding. The first level of protection is a set of ionization chambers mounted on the beam lines that are connected to the Fast Protect System. They are set to trip at low levels and are most often used during beam tuning. This system automatically resets, without operator intervention, once a fault condition has cleared. The next level of protection, the Run Permit System, utilizes a different set of ionization chambers near the beam lines as well as neutron detectors outside the shielding. If the Run Permit System trips, it must be reset by the accelerator operator. The final level of ARMD is the Radiation Security System. It is a safety rated, redundant, and fail-safe system, which uses strategically placed ionization chambers (in pairs) inside the beam tunnels. If the Radiation Security System trips, the other two ARMD systems will also trip and the redundant fusible beam plugs will be inserted in the low-energy transport region of the LINAC. Administrative procedures require management approval before beam operations can resume after a Radiation Security System fault.

\section{$\underline{\text { TRIUMF }}$}

An interlocked system of 25 neutron detectors (trip level at $1 \mathrm{mSv} \mathrm{h}^{-1}$ and warning level at 0.01 $\mathrm{mSv} \mathrm{h}^{-1}$ ) is installed outside the shielding. Most are located over beamlines where an abnormal beam loss would cause significant fields outside shielding. The 'trip' setpoint for all detectors is $1 \mathrm{mSv} \mathrm{h}^{-1}$. The 'time-to-trip' is also $200 \mathrm{msec}$.

\section{$\underline{\text { TJNAF }}$}

The ARMDs (known as Controlled Area Radiation Monitor at TJNAF) are located in controlled areas. Each ARMD continuously monitors and records neutron and photon dose equivalent rates. The ARMDs are not considered as one of the "three independent means employing at least two different technologies" that are called for in this policy. Rather, it is to be regarded as an additional level of protection above and beyond that required.

\section{DFELL}

Photon detectors are located around the facility. They are set to trip at $0.05 \mathrm{mSv} \mathrm{h}^{-1}$ (killing the high voltage to the Linac RF drivers) and to warn at $0.02 \mathrm{mSv} \mathrm{h}^{-1}$. Each detector is self-tested by a builtin photon source. 


\section{CONCLUSIONS}

Radiation safety problems at accelerator facilities are different from those of nuclear facilities, especially in the protection against the prompt radiation hazards. Currently there are no detailed standards or guidelines for the establishment of radiation safety systems. Using several North American accelerator facilities as examples, the radiation protection policies and practices at these facilities are illustrated and compared. It is hoped that this overview will assist in having a sound, consistent and harmonized approach in establishing radiation safety systems amongst the accelerator community.

\section{GLOSSARY}

ACS: Access Control System

ARMD: Active Radiation Monitoring Device

BSY: Beam Switch Yard

DFELL: Duke Free Electron Laser Facility

HENIT: High-Energy North Injection Transport line

HER: High-Energy storage Ring

IR: Interaction Region

LANSCE: Los Alamos Neutron Science Center

LER: Low-Energy storage Ring

LESIT: Low-Energy South Injection Transport line

PPS: Personnel Protection System

RCS: Radiation Containment System

RSS: Radiation Safety System

SLAC: Stanford Linear Accelerator Center

SSRL: Stanford Synchrotron Radiation Laboratory

TJNAF: Thomas Jefferson National Accelerator Facility

TRIUMF: Tri-University Meson Facility 


\section{REFERENCES}

1. Liu, J. C., Bong, P., Gray, B., Mao, X. S., Nelson, G., Nelson, W. R., Schultz, D. and Seeman, J. Radiation Safety System of the B-Factory at the Stanford Linear Accelerator Center, Health Phys. 77(5), 588-594 (1999).

2. Liu, J. C., Ipe, N. E. and Yotam, R. Radiation Safety System for Stanford Synchrotron Radiation Laboratory, Proceedings of the International Workshop on Radiation Safety at Synchrotron Radiation Sources, Argonne National Laboratory, Argonne, IL, April 23-24 (2001).

3. Rokni, S. H., et al. Radiation Protection Systems for the Final Focus test Beam at SLAC, Health Phys. 71(5), 786-794 (1996).

4. Ipe, N. E. and Liu, J. C. Shielding and Radiation Protection at the SSRL 3 GeV Injector, Proceedings of American Nuclear Society Topical Meeting, New Horizons in Radiation Protection and Shielding, Pasco, Washington, April 26 - May 1, (1992).

5. Yotam, R. et al., Personnel Protection and Beam Containment Systems for the 3-GeV Injector, Proceedings of the IEEE 1991 Particle Accelerator Conference, San Francisco, CA (1991).

6. Gallegos, F., LANSCE Radiation Security System (RSS), Proceedings of the Midyear Topical Meeting of the Health Physics Society, San Jose, California, 5-8 Jan (1996).

7. Drozdoff, J. The Radiation Safety System at TRIUMF, TRIUMF, Vancouver, Canada, TRIUMF Internal Report (Draft) (2001).

8. TJNAF, Jefferson Lab Beam Containment Policy, TJNAF, Newport News, VA, http://www.jlab.org/ehs/manual/EHSbook-403.html, October (1994).

9. 10CFR835, Title 10 Code of Federal Regulations Part 835, Occupational Radiation Protection (1998).

10. National Council on Radiation Protection (NCRP) Radiation Alarms and Access Control Systems, NCRP, Bethesda, MD, NCRP Report No. 88 (1986). 
Captions of Figures and Tables

Figure 1. Schematic layout of the B-Factory accelerator facility and its related beam stopper systems. The power limiting devices (average current monitors and meter relays for bends in this case) and the devices to protect the beam stoppers are also shown.

Figure 2. Schematic layout of the Los Alamos Neutron Science Center (LANSCE) accelerators and its safety systems.

Figure 3. Schematic layout of the TRIUMF cyclotron vault and its beam inhibiting devices in each main beamline.

Figure 4. Schematic layout of the TJNAF facility (injector, Linac, switch yard, and three experimental halls).

Figure 5. Illustration of the Access Control System (ACS) and Radiation Containment System (RCS) at SLAC. Typical features of the entry module for the ACS are also shown.

Figure 6. The beam stopper systems at the SSRL, a synchrotron radiation accelerator facility at SLAC (see text for detail).

Table 1. Three levels of injection beam power, normal, allowed and maximum credible beam (two levels for stored beam energy), to be considered in shielding design for the B-Factory. 


\begin{tabular}{lllll}
\hline \multirow{2}{*}{ Level } & \multicolumn{2}{l}{ Injection Beam Power $(\mathrm{kW})$} & \multicolumn{2}{l}{ Stored Beam Energy $(\mathrm{kJ})$} \\
\cline { 2 - 5 } & Electron & Positron & Electron & Positron \\
\hline Normal Beam $^{\mathrm{a}}$ & 1.2 & 0.22 & $67(1 \mathrm{~A})$ & $49(2.1 \mathrm{~A})$ \\
Allowed Beam $^{\mathrm{b}}$ & 3.5 & 1.5 & $269(3 \mathrm{~A})$ & $90(3 \mathrm{~A})$ \\
Maximum Credible Beam $^{\mathrm{b}}$ & 333 & 10 & $\mathrm{NA}$ & $\mathrm{NA}$ \\
\hline
\end{tabular}

${ }^{a}$ Energy: $9 \mathrm{GeV}$ electron and $3.1 \mathrm{GeV}$ positron.

${ }^{\mathrm{b}}$ Energy: $12 \mathrm{GeV}$ electron and $4 \mathrm{GeV}$ positron. 


\section{RSS for B-Factory}

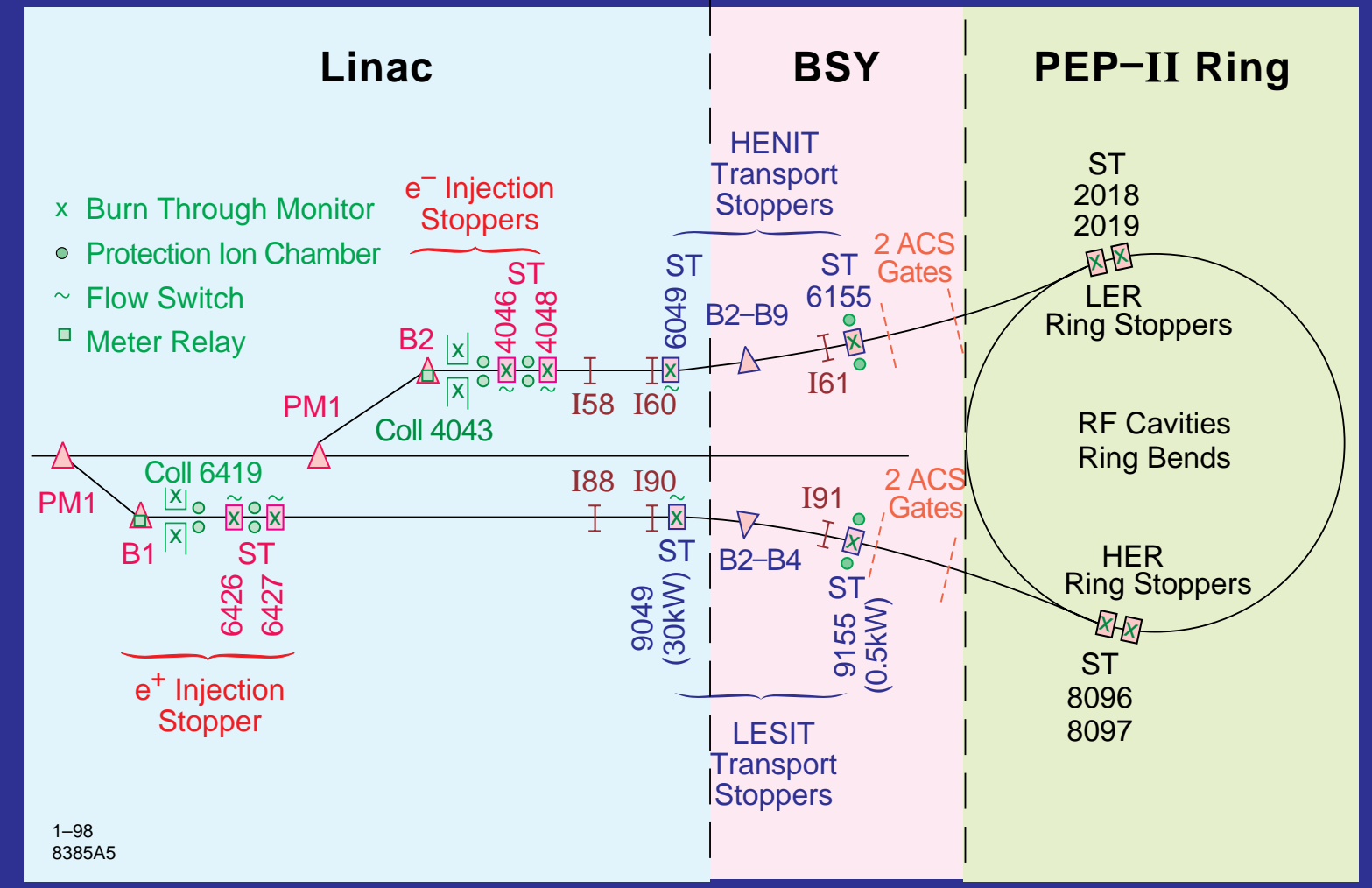




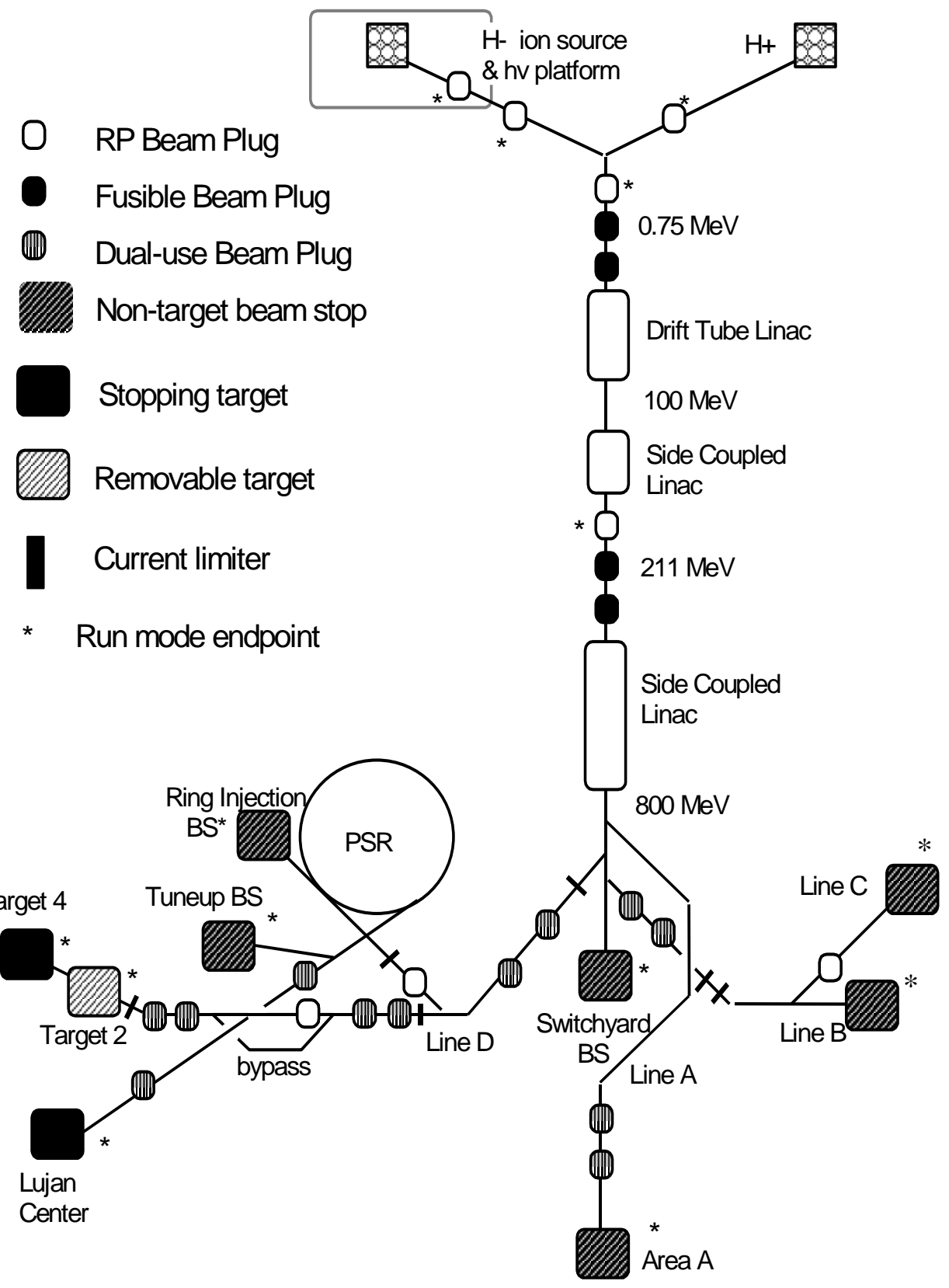




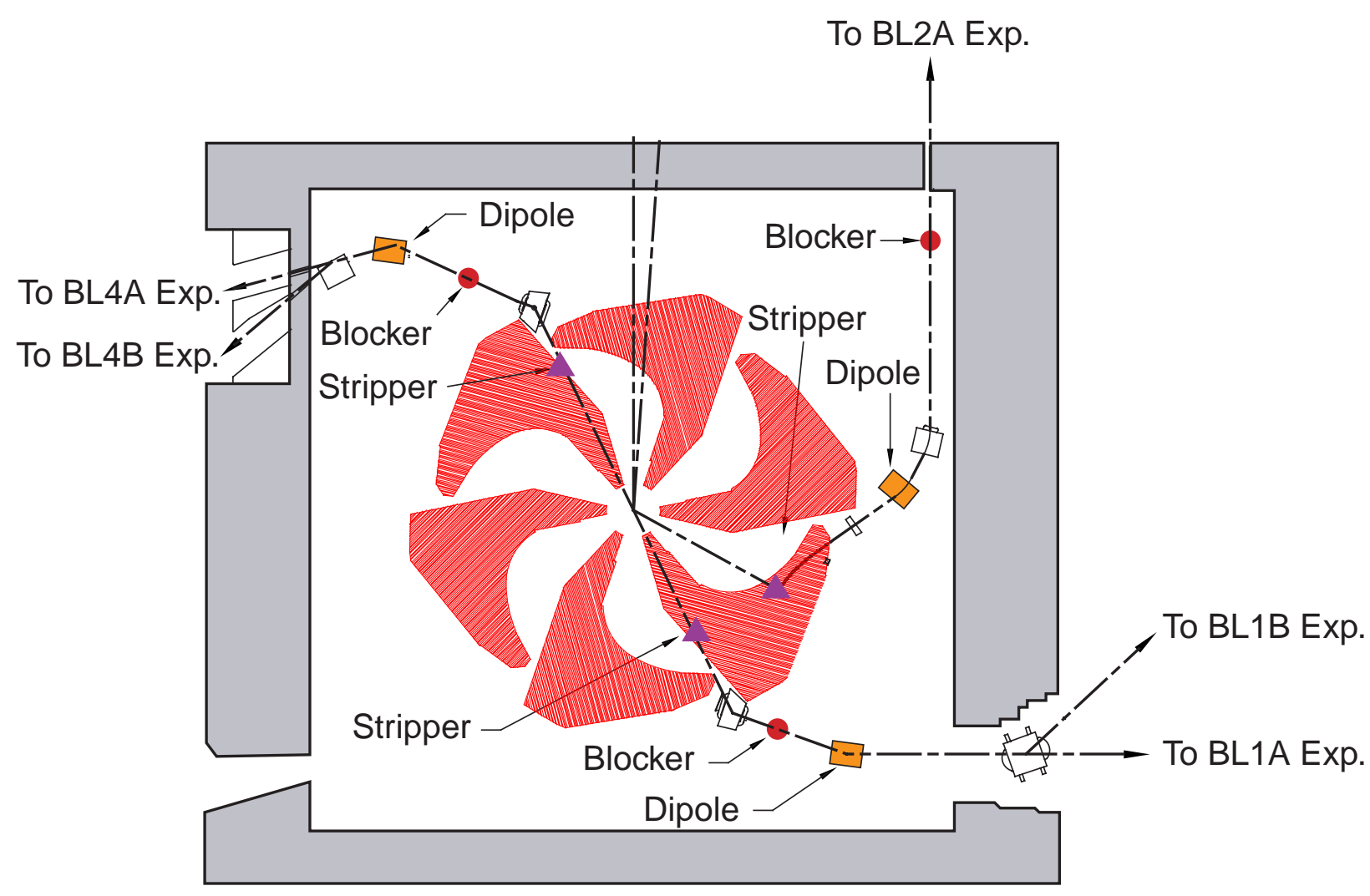

TRIUMF Cyclotron Vault 


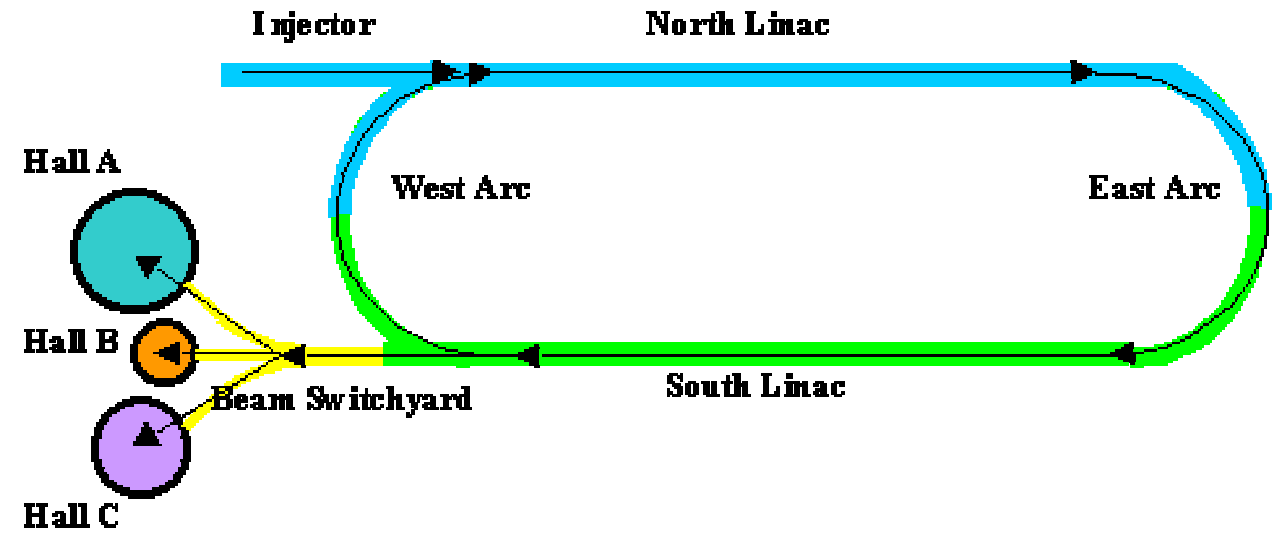

CEBAF Layout at Jefferson Lab 


\section{A Schematic Layout of ACS \& RCS}

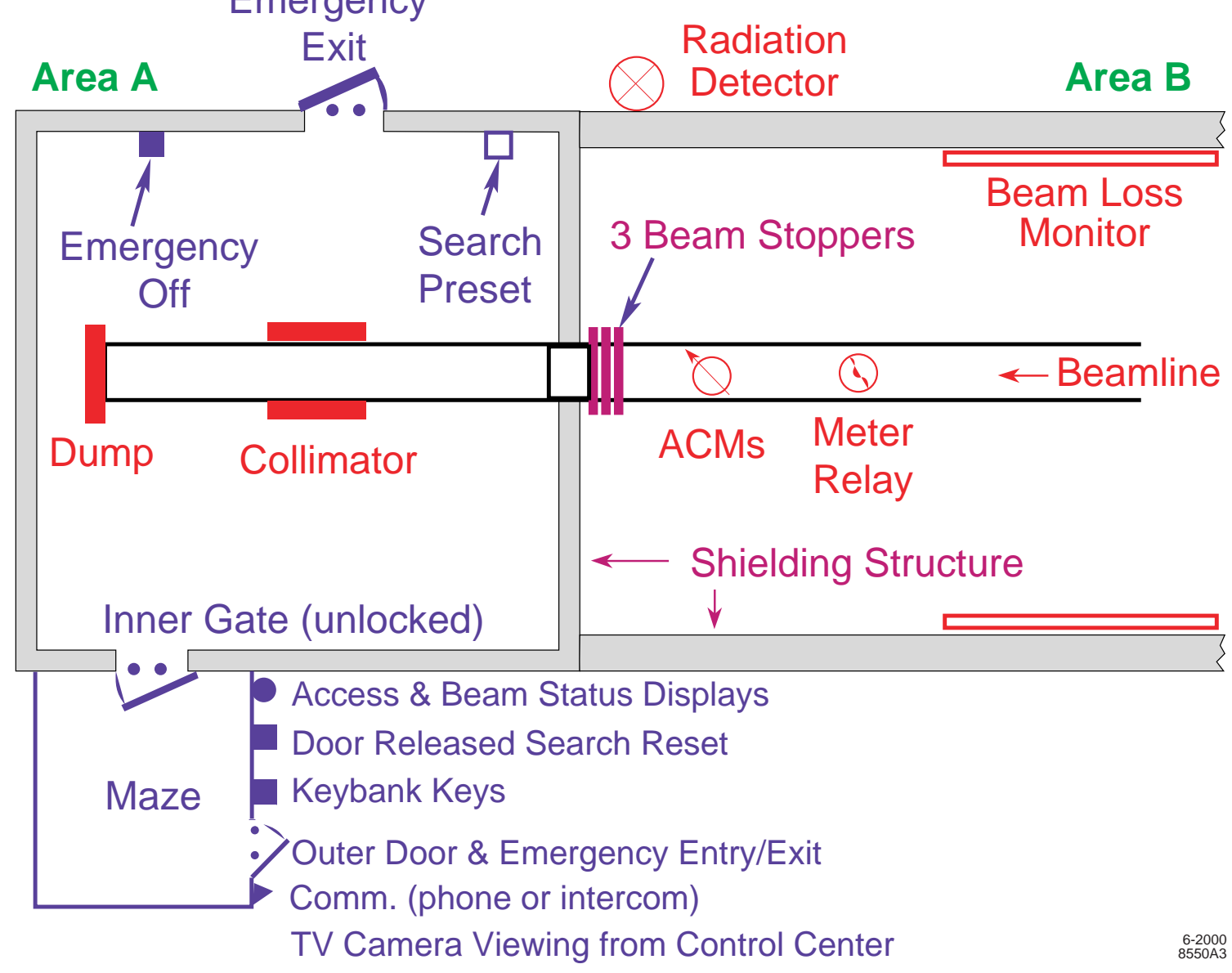




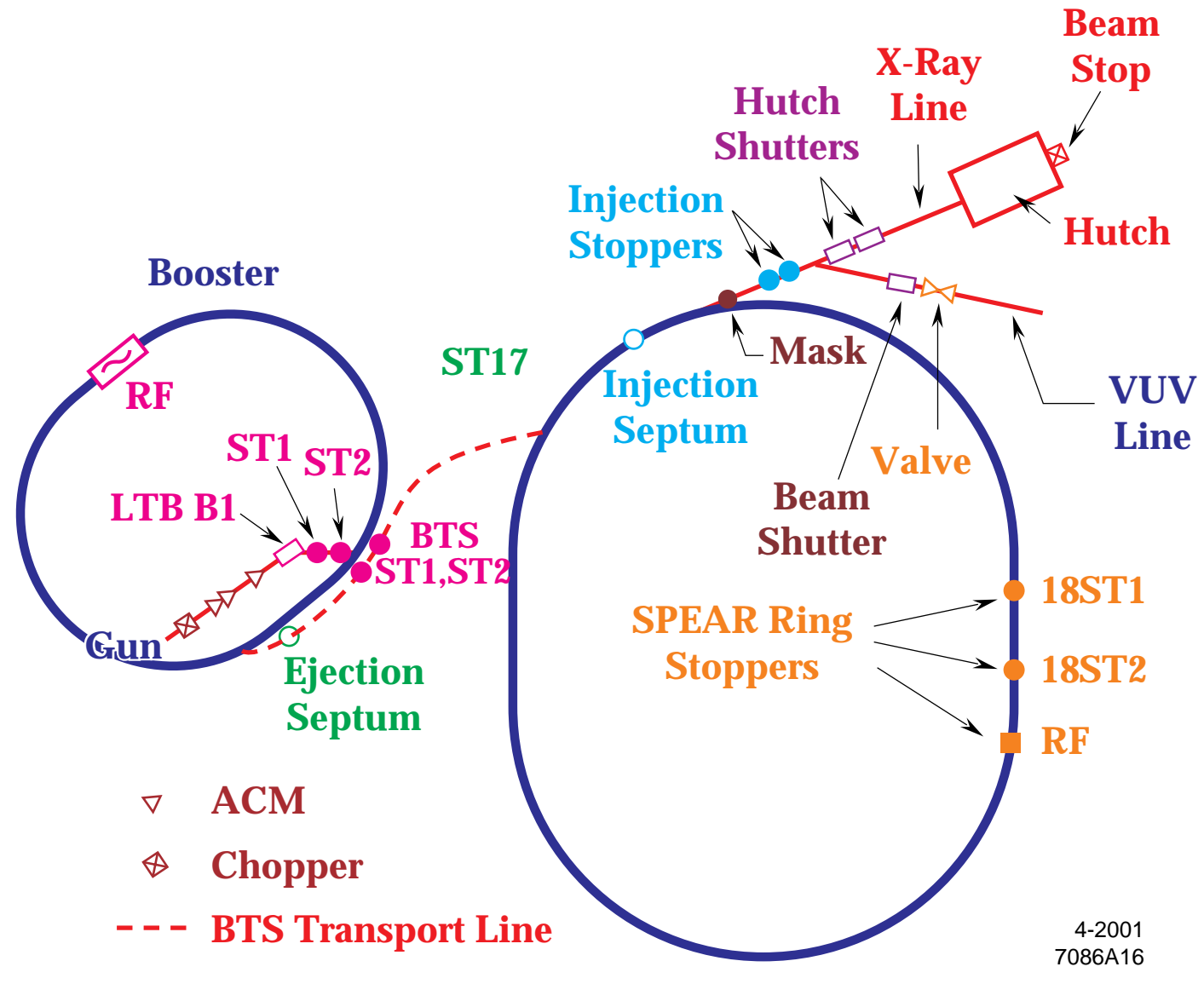

